\title{
Oncoproteomics: New trends in analytical techniques
}

\author{
Girish Gupta ${ }^{1}$, Nikhil Gupta ${ }^{2}$,Mayank Gupta ${ }^{2}$, Soham Trivedi ${ }^{3}$, Prasad Patil ${ }^{4}$, \\ Vamsi Krishna $K^{5}$, Hipal Gaudani ${ }^{3}$ and Gomase V.S. ${ }^{6}$ \\ ${ }^{1}$ S.D.S.M. College Palghar, Mumbai \\ ${ }^{2}$ Sindhu Mahavidyalaya Panchpaoli Nagpur \\ ${ }^{3}$ V.V.P. Engineering College, Rajkot, Gujrat \\ ${ }^{4}$ Dr. D.Y. Patil ACS College, Pimpri, Pune \\ ${ }^{5}$ Rai foundations College CBD Belapur Navi Mumbai \\ ${ }^{6}$ School of Technology, S.R.T.M. University, Sub-Centre, Latur, 413512, India
}

\begin{abstract}
Oncoproteomics is the study of proteins and their interactions in a cancer cell by proteomic technologies and has the potential to revolutionize clinical practice, including cancer diagnosis. Oncoproteomics screening based on proteomic platforms as a complement to histopathology, individualized selection of therapeutic combinations that target the entire cancer-specific protein network, real-time assessment of therapeutic efficacy and toxicity and rational modulation of therapy based on changes in the cancer protein network associated with prognosis and drug resistance. Oncoproteomics refers to the application of proteomic technologies in oncology and parallels the related field of oncogenomics. The challenges ahead and perspectives of oncoproteomics for biomarkers development are also addressed. With a wealth of information that can be applied to a broad spectrum of biomarker research projects serves as a reference for biomarker researchers, scientists working in proteomics and bioinformatics, oncologists, pharmaceutical scientists, biochemists, biologists, and chemists.
\end{abstract}

Keywords- Oncoproteomics, Genomics, Proteomics, NMR, MS, GC

\section{Introduction}

Disease incidence of cancer and its associated mortality are increasing globally, indicating an urgent need to develop even more effective and sensitive sets of biomarkers that could help in early diagnosis and consequent intervention [1]. Oncoproteomics is the study of proteins and their interactions in a cancer cell by proteomic technologies and has the potential to revolutionize clinical practice, including cancer diagnosis and screening based on proteomic platforms as a complement to histopathology, individualized selection of therapeutic combinations that target the entire cancerspecific protein network, real-time assessment of therapeutic efficacy and toxicity, and rational modulation of therapy based on changes in the cancer protein network associated with prognosis and drug resistance. Technology is also applied to the discovery of new therapeutic targets and to the study of drug effects. The study of oncoproteomics provides mankind with a better understanding of neoplasia [2]. Proteomic research first came to the fore with the introduction of two-dimensional gel electrophoresis. At the turn of the century, proteomics has been increasingly applied to cancer research with the wide-spread introduction of mass spectrometry and proteinchip [3]. Many myriad processes are involved in the diseased states; the goal is similar to 'finding a needle in a haystack'. Also, the development of new 'omics' technologies; such as genomics and proteomics, has allowed us to monitor a large number of key cellular pathways simultaneously $[11,15]$. Proteomics is a promising approach in the identification of proteins and biochemical pathways involved in carcinogenesis. Proteomic technologies are now being incorporated in oncology in the postgenomic era [20]. Oncoproteomics is playing an increasingly important role in the diagnosis and management of cancer as well as in the development of personalized treatment of carcinomas. Innovative proteomic technologies relevant to cancer are described briefly, which are helping in the understanding of mechanism of drug resistance in cancer and will provide some leads to improve the management [25]. Oncoproteomics refers to the application of proteomic technologies in oncology and parallels the related field of oncogenomics. Use of proteomic technologies for study of cancer is well established. Some innovative technologies used for study of molecular biology of cancer, detection of cancer biomarkers and their application in molecular diagnosis of cancer as well as anticancer drug discovery. Some of the challenges will be identified and finally the role of oncoproteomics in the development of personalized cancer therapy [14]. New research in postgenome era, proteomics provides a powerful approach for the analysis of normal and transformed cell functions, for the identification of disease-specific targets, and for uncovering novel endpoints for the evaluation of chemoprevention agents and drug toxicity [30]. The current advances in genomic and proteomic laboratory technology which is critically appraise those studies, which have utilized these latest technologies and ascertain their potential to identify clinically useful biomarkers [22]. Hepatocellular carcinoma (HCC) is a heterogeneous cancer with no promising 
treatment and remains one of the most prevailing and lethal malignancies in the world as for the cancer treatments. Scientists in many biological areas now routinely identify and characterize protein markers by a mass spectrometry-based proteomic approach, a method that has been commonly used to discover diagnostic biomarkers for cancer detection [17]. Oncoproteomics is the application of proteomics technologies in oncology; functional proteomics is a promising technique for the rational identification of biomarkers and novel therapeutic targets for cancers. Omics progress in proteomics has opened new avenues for tumorassociated biomarker discovery; with the advent of new and improved proteomics technologies, such as the development of quantitative proteomic methods, high-resolution, -speed and sensitivity mass spectrometry and protein arrays, as well as advanced bioinformatics for data handling and interpretation, it is now possible to discover biomarkers that can reliably and accurately predict outcomes during cancer management and treatment [28].

\section{History and Background}

Oncoproteomics is the study of proteins and their interactions in a cancer cell by proteomic technologies. Proteomic research first came to the fore with the introduction of two-dimensional gel electrophoresis. At the turn of the century, proteomics has been increasingly applied to cancer research with the wide-spread introduction of mass spectrometry and proteinchip. There is an intense interest in applying proteomics to foster an improved understanding of cancer pathogenesis, develop new tumor biomarkers for diagnosis, and early detection using proteomic portrait of samples [2]. Oncoproteomics has the potential to revolutionize clinical practices, including cancer diagnosis, treatments and useful for screening based on proteomic platforms as a complement to histopathology, individualized selection of therapeutic combinations that target the entire cancer-specific protein network, real-time assessment of therapeutic efficacy and toxicity. Oncoproteomics also applicable in rational modulation of therapy based on changes in the cancer protein network associated with prognosis and drug resistance. However, oncoproteomics is also applied to the discovery of new therapeutic targets and to the study of drug effects. The challenges ahead and perspectives of oncoproteomics for biomarkers development are also addressed. With a wealth of information that can be applied to a broad spectrum of biomarker research projects serves as a reference for biomarker researchers, scientists working in proteomics and bioinformatics, oncologists, pharmaceutical scientists, biochemists, biologists, and chemists [2]. One potential solution to finding cancer-associated protein signatures is the emerging technology of affinity proteomics. This approach addresses some of the shortcomings of traditional proteomics and combines it with the power of microarrays. The present review focuses on the role of antibody microarrays in oncoproteomics and its potential to provide a truly proteome-wide analytical approach [5]. The term cancer proteome refers to the collection of proteins expressed by a given cancer cell and should be considered as a highly dynamic entity within the cell, which affects a variety of cellular activities during cancerous stages. The emerging proteomic analysis platforms including 2D-PAGE, mass spectrometry technologies, and protein microarrays represent powerful tools to study and understand cancer [16].

\section{Methodology-Analytical techniques}

Oncoproteomics encompasses the identification and quantitative analysis of the entire complement of protein in a biological samples and commonly used technologies available today include 2D-polyarcylamide gel electrophoresis (2$\mathrm{DE})$, isotope-coded affinity tags (ICAT), matrix assisted laser desorption ionization-mass spectrometry (MALDI-MS), liquid chromatography-MS/MS (LC-MS/MS), imaging MS, protein arrays and autoantibody expression techniques [19].

\section{Mass Spectrometry (MS)}

Mass spectrometry (MS) has become the analytical techniques of choice in proteomic study owing to its quantitative capability and facility to interface with the different chromatographic separation methods [19]. Liquid chromatography-mass spectrometry (LC-MS) profiling of clinical samples for quantifying absolute ion abundances of peptides and proteins has emerged as a promising approach. Quantitation of changes in protein abundance of large number of samples is challenging and requires automatic processing means. The development of data analysis software is laborious and time-consuming. Freely available tools have been recently introduced, which incorporate algorithms for visualization and data processing and allow the user to embed external routines for data analysis. A relevant issue related to the design and evaluation of such tools concerns usability [26]. Mass spectrometry analysis driven in quantitative multiple reactions monitoring (MRM) mode is now appearing as a promising alternative to quantify proteins in biological fluids. In MRM, MS analysis time is focused only on analyte of specific masses, while all others are excluded. The application of MRM to proteomic analysis has been adopted because of advances in MS instrumentation. These studies highlighted fibulin-2 as a breast cancer 
marker in mice, and CEA as a lung cancer marker. Its application to $\mathrm{CRC}$ has yet to produce a definite potential biomarker. In an AQUA (Absolute quantification) study, a peptide containing a stable-isotope labeled amino acid is developed based on the sequence of a peptide, which is being targeted for quantitation and for validation of biomarkers tends to be less time consuming than MS-based quantitation of peptides [19]. Multidimensional LC-MS/MS has been used for the analysis of biological samples labeled with isobaric mass tags for relative and absolute quantitation (iTRAQ) to identify proteins that are differentially expressed in human headand-neck squamous cell carcinomas (HNSCCs) in relation to non-cancerous head-and-neck tissues (controls) for cancer biomarker discovery.

\section{Role of Mass Spectrometry in Proteomics}

The total proteome consists of well over a million different protein species and that the dynamic range of expression of proteins varies over 108 to 109 orders of magnitude i.e., some estimates in serum are up to 1012. Some proteins or modified forms of proteins may be expressed during very brief periods during the life of an individual, for example during embryonic development, whereas others may be continually expressed at very low levels (a few copies per cell). MS has become an indispensable tool for proteomic studies for the detection, identification, and characterization of the protein component of cells, tissues, and organs at any point in health and disease. In proteomics analysis, several types of instruments and protocols allow the determination of molecular weight, primary and higher order structure, post-translational modifications, quantification, and localization. Desorption ionization techniques such as MALDI-mass spectrometry (MALDI-MS) and electrospray ionization (ESI) have revolutionized our ability to analyze large biomolecules, including peptides and intact proteins, with unsurpassed sensitivity, resolution and mass accuracy. It is possible to routinely measure molecular weights above 200 kD, obtain accurate, low parts-per-million (ppm) mass measurements on peptides and proteins, fragment individual peptides using tandem mass spectrometry for protein identification, and characterize and map sites of post-translational modifications [19].

\section{Two Dimensional Electrophoresis: 2-DE}

The study shows that expression of secretagogin in non-neuronal and non-neuroendocrine cells may represent aberrant expression of the protein and may be related to de- or trans-differentiation phenomena. The down-regulation of secretagogin, a protein expressed in neuroendocrine cells of the colonic crypts in carcinomatous mucosal cells involved in calcium- binding and was an invitro study using immunohistochemistry. So further in vivo studies are needed for it to progress to a clinical setting; it has certainly been highlighted as a potential for the future. Not alone implicated in CRC, secretagogin expression is currently under scrutiny in several tumour types, with recent researches examining its role in prostatic adenocarcinoma, pituitary adenomas, carcinoid tumours and their metastases as well as neuroendocrine tumours from the lung, pancreas and adrenal gland [19].

\section{Protein microarrays: new tools for pharmaceutical development}

Protein microarrays are a relatively new technology, which will dramatically impact the pharmaceutical industry and applicable for validation of new target for drug developments. The critical need for more rapid identification of novel drug targets and for obtaining high-quality information early in the target validation process is a major driver for the industry. High-throughput microarray technology platforms allow for simultaneous, multi-parametric analysis of complex protein mixtures. Protein microarrays have tremendous potential as a tool for the study of protein-protein, enzyme-substrate, and antibody-antigen interactions among others. They can also be used for biomarkers and drug target identification via comparative proteomic analysis of healthy and disease tissues [3]. Antibody microarrays have great potential for significant value in biological research and experimental formats can be broadly categorized into two classes: (i) direct labeling experiments, and (ii) dual antibody sandwich assays. In the direct labeling method, the covalent labeling of all proteins in a complex mixture provides a means for detecting bound proteins after incubation on an antibody microarray. If some proteins are labeled with a tag, such as biotin, the signal from bound proteins can be amplified. In the sandwich assay, proteins captured on an antibody microarray are detected by a cocktail of detection antibodies, each antibody matched to one of the spotted antibodies. Several applications of antibody arrays to cancer research have been reported, including the analysis of proteins in blood serum, resected frozen tumors, cell lines, and on membranes of blood cells [4]. The microarray patterns that are generated can then be converted into proteomic maps, or molecular fingerprints, revealing the composition of the proteome. Using this techniques, global proteome analysis and protein expression profiling will thus provide new opportunities for biomarker discovery, drug target identification and disease diagnostics, as well as providing insights into disease biology [13]. 


\section{MALDI-TOF}

Matrix-Assisted Laser Desorption/lonization Time of Flight technique (MALDI-TOF) techniques are use to be analysed is mixed with an energy absorbing matrix molecule, which absorbs light at a predetermined wavelength. MALDI-TOF technology is also being applied in the search to predict metastasis in known cases of CRC. Two CRC cell lines with different metastatic potentials, SW480 and SW620, were recently investigated using MALDI-TOF to search for potential markers for predicting CRC metastasis [19].

\section{SELDI-TOF}

Surface enhanced laser desorption ionization/time of flight (SELDI-TOF) is a new techniques of complex protein lysis based on MALDI technology. SELDI mass spectrometry is used to investigate protein expression in sera of patients with gastric cancer and gastritis compared to normal volunteers. The first SELDITOF study attempted to differentiate CRC patients from those with colorectal adenoma. SELDI-TOF technology is has recently been applied to the identification of responders and non-responders to neo adjuvant chemoradiotherapy (RCT). SELDI techniques used for the generation of proteomic patterns in order to identify potential biomarkers associated with the diagnosis and prognosis of a variety of cancers [24]. A new mass spectrometric technology involves surface enhanced laser desorption ionization combined with time-of flight mass analysis (SELDI-TOF-MS), using special protein chips. The scenario of the relevant principles of the technique, including approaches to proteomic pattern diagnostics, applications are reviewed for the diagnosis of ovarian, breast, prostate, bladder, pancreatic, and head and neck cancers, and also several other malignancies [27].

\section{Laser Capture Microdissection}

Laser capture microdissection (LCM) provides an ideal method for extraction of cells from specimens, which the exact morphologies of both the captured cells and the surrounding tissue are preserved. LCM is compatible with subsequent nucleic acid analysis of tissues and is important for comprehensive molecular characterization of normal, precancerous, and malignant cells by means of DNA-array technology.

\section{Current research scenario}

Oncoproteomics is the application of proteomics technologies in oncology and functional proteomics is a promising technique for the rational identification of biomarkers and novel therapeutic targets for melanomas. Recent progress in proteomics has opened new avenues for tumor-associated biomarker discovery. With the advent of new and improved proteomics technologies, such as the development of quantitative proteomic methods, high-resolution, speed and sensitivity mass spectrometry and protein arrays, as well as advanced bioinformatics for data handling and interpretation, it is now possible to discover biomarkers that can reliably and accurately predict outcomes during cancer management and treatment. Also, there are several difficulties in the study of proteins/peptides, which are not inherent in the study of nucleic acids. New challenges arise in large-scale proteomic profiling when dealing with complex biological mixtures. Nevertheless, oncoproteomics offers great promise for unveiling the complex molecular events of tumorigenesis, as well as those that control clinically important tumor behaviors,

such as metastasis, invasion and resistance to therapy [19]. Proteomics has been applied in anticancer drug discovery and for personalized management of cancer. Proteins can be identified from the blood or directly from the tumor tissue by laser capture microdissection (LCM) and tissue microarrays. Protein biochips can be used for diagnosis as well as monitoring in clinical trials. Current research in nanobiotechnology has refined the use of proteomics and nanoproteomics and has improved most current protocols including protein purification / display and automated identification of protein traces in minute samples. Proteomic studies through light tumor invasiveness and drug resistance. Examples of applications of oncoproteomics are given for cancers of various organs such as the brain, breast, colon and rectum, prostate, and leukemia. In conclusion, proteomics will play an important role in diagnosing cancer and developing personalized treatment of cancer [29]. Advances in proteomics are contributing to the understanding of pathophysiology of cancer, cancer diagnosis and anticancer drug discovery. Laser capture microdissection (LCM) provides an ideal method for extraction of cells from specimens in which the exact morphologies of both the captured cells and the surrounding tissue are preserved. Differentially xpressed proteins in tumor tissue are found by comparing the protein expression patterns generated using SELDI (surface-enhanced laser desorption / ionization)-based protein chip technology. Oncoproteomics technologies have been used for the study of cancer of various organs. Continued refinement of techniques and methods to determine the abundance and status of proteins in vivo holds great promise for future study of cancer and development of personalized cancer therapies [21]. 


\section{Conclusion}

The technical aspects and challenges in the development and uses of antibody microarray assay and examine recently reported applications in oncoproteomics. The use of antigen and antibody arrays could revolutionize the area of biomarker discovery, deciphering biochemical and signaling pathways and pharmaceutical research. The ProteinChip Array technology forms the basis of a clinical proteomics platform designed to expedite the discovery, validation, and characterization of cancer biomarkers at all stages of cancer progression. An immediate goal of proteomic studies is the understanding of proteins including their expression, function, interaction and structure with an endpoint of discovery of protein biomarkers. Such biomarkers can be used in detection, prognostication and treatment of many diseases. A new subdiscipline named clinical proteomics, concomitant with new molecular technologies, which are developed, demonstrates promise to discover new cancer biomarkers. Current research in diagnosis of cancer, even in a premalignant state, is crucial for the successful treatment of this disease. For these reasons, it is clear that the identification of biomarkers for the early diagnosis of cancer should represent one of the main goals of this emerging field of study. Gene expression profile is important for personalising cancer therapy. Molecular imaging, such as by positron emission tomography, enables determination of tumour response to drug action at the molecular level; the combination of diagnostics with therapeutics-an important feature of personalised cancer therapy--is facilitated by the use of monoclonal antibodies and nanobiotechnology. New laboratory techniques of protein identification may eventually allow identification of a clinically useful biomarker, which could be used for screening, validation and treatment.

\section{Reference}

[1] Alhamdani M.S., Schroder C., Hoheisel J.D. (2009) Genome Med., 1(7),68.

[2] Cho W.C. (2007) Mol Cancer. 2; 6:25.

[3] Kumble K.D. (2003) Anal Bioanal Chem. $377(5): 812-9$

[4] Haab B.B. (2003) Proteomics. 3(11):211622.s

[5] Borrebaeck C.A. (2006) Expert Opin Biol Ther. 6(8):833-8.

[6] Mafcgregor P.F. (2003) Expert Rev Mol Diagn. 3(2):185-200.

[7] Wingren C., Borrebaeck C.A. (2007) Expert Rev Mol Diagn. 7(5):673-86.

[8] Sreekumar A., Chinnaiyan A.M. (2002) Curr Opin Mol Ther. 4(6):587-93.

[9] Yip T.T., Lomas L. (2002) Technol Cancer Res Treat. 1(4):273-80.
[10] Ramon Aragues, Chris Sander and Baldo Oliva. (2008) BMC Bioinformatics. 9:172.

[11] Kumar S., Mohan A. and Guleria R. (2006) Biomarkers. 11(5):385-405.

[12] Aebersold R., Auffray C., Baney E., Barillot E., Brazma A., Brett C., Brunak S., Butte A., Califano A., Celis J., Cufer T., Ferrell J., Galas D., Gallahan D., Gatenby R., Goldbeter A., Hace N., Henney A., Hood L., lyengar R., Jackson V., Kallioniemi O., Klingmüller U., Kolar P., Kolch W., Kyriakopoulou C., Laplace F., Lehrach H., Marcus F., Matrisian L., Nolan G., Pelkmans L., Potti A., Sander C., Seljak M., Singer D., Sorger P., Stunnenberg H., SupertiFurga G., Uhlen M., Vidal M., Weinstein J., Wigle D., Williams M., Wolkenhauer O., Zhivotovsky B., Zinovyev A., Zupan B. (2009) Mol Oncol. 3(1):9-17.

[13] Wingren C., Borrebaeck C.A. (2006) OMICS. 10(3):411-27.

[14] DeVita V.T. Jr., Bleickardt E.W. (2001) Cancer J. 7 Suppl 1:S2-13.

[15] Gagnon A., Ye B. (2008) Curr Opin Obstet Gynecol. 20(1):9-13.

[16] Alessandro R., Fontana S., Kohn E., De Leo G. (2005) Tumori. 91(6):447-55

[17] Sun S., Lee N.P., Poon R.T., Fan S.T., He Q.Y., Lau G.K., Luk J.M. (2007) Liver Int. 27(8):1021-38.

[18] Jain K.K. (2005) Expert Opin Pharmacother. 6(9):1463-76

[19] Jain K.K. (2004) Expert Rev Proteomics. $1(1): 49-55$.

[20] Jain K.K. (2002) Technol Cancer Res Treat. $1(4): 219-20$.

[21] Jain K.K. (2002) Curr Opin Mol Ther. 4(3):203-9.

[22] McHugh S.M., O'Donnell J., Gillen P. (2009) World J Surg Oncol. 7:36.

[23] Alessandro R., Fontana S., Kohn E., De Leo G. (2005) Tumori.91(6):447-55.

[24] Espejel F., Roa J.C. (2008) Med Clin (Barc). $131(8): 312-7$.

[25] Jain K.K. (2008) Mol Oncol. 2(2):153-60.

[26] Matta A., DeSouza L.V, Shukla N.K, Gupta S.D., Ralhan R., Siu K.W. (2008) J Proteome Res. 7(5):2078-87.

[27] Roboz J. (2005) Cancer Invest. 23(5):46578.

[28] Cho W.C, Cheng CH. (2007) Expert Rev Proteomics. 4(3):401-10.

[29] Jain K.K. (2007) J Buon. 12 Suppl 1:S31-8.

[30] Verma M., Wright G.L. Jr., Hanash S.M., Gopal-Srivastava R., Srivastava S. (2001) Ann N Y Acad Sci., 945:103-15. 